\title{
Gaming to Learn astronomy, an innovation approach, two study cases
}

\author{
Margarita Metaxa ${ }^{1}$ \\ ${ }^{1}$ Philekpaideutiki Etaireia, and National Observatory of Athens, Greece
}

\begin{abstract}
In this paper I am going to present you two innovative approaches for learning Astronomy through gaming. On one hand we used the subject of Astronomy as a vehicle since Astronomy provides a unique environment for educators from Kindergarten to Lyceum, and because its multidisciplinary character is ideal for an introduction to science education. On the other hand Gaming to learn has been around for a decade, but it is only recently that these possibilities in the realm of education truly have been appreciated. If indeed humans think immeasurably better as part of a network than on their own, then games are an obvious terrain in which to set minds free and let them wander around. Thus it;s really worthy to test and try this new medium of learning to a fachinating science.
\end{abstract}

\section{Introduction}

We believe that by developing and promoting the teaching of Astronomy in the broadest possible way to students we can introduce students to science in a very pleasant way and easily prepare them for a "life- long learning" journey ${ }^{[3]}$. Furthermore, the cultural and philosophical role of Astronomy is undisputed. Studying the Universe is a way of searching for our own origin, learning to situate ourselves within cosmic infinity and developing a sense for the beauty and fragility of our planet the Earth. It also allows us to keep a critical approach towards irrational pseudo-sciences ${ }^{[4]}$.

Thus it's a unique opportunity to involve Astronomy to the new case for Educational Learning through Play. Those who believe in using games in education usually start from a common set of assumptions. They observe that game players regularly exhibit persistence, risk-taking, attention to details and problem solving skills, all behaviors that ideally would be regularly demonstrated in school. They also understand that game environments enable players to construct understanding actively, and at individual paces, and that well-designed games enable players to advance on different paths at different rates in response to each player's interests and abilities, while also fostering collaboration and just-in-time learning i.e. «collective intelligence» ${ }^{[5]}$.

In other words games which are formalized expressions of play allowing people to go beyond immediate imagination and direct physical activity, help students to develop noncognitive skills that are as fundamental as cognitive skills in explaining how we learn and if we succeed. Skills such as patience and discipline, which one should acquire as a child but often does not, correlate with success better than IQ scores do ${ }^{[6]}$. And those non-cognitive skills - that is, not what you know but how you behave - are far better suited to a game context than to a traditional classroom and textbook context.

Gamification, is the application of game-design elements and game principles in nongame contexts to improve user engagement, organizational productivity, flow, learning, and more. 


\section{History of games}

Games are an integral part of all cultures and are one of the oldest forms of human social interaction (eg. games are found in burials in Egypt 3100 BC, in prehistoric India, Plato and Homer mention board games called 'petteia, etc). According to the German philosopher Friedrich Schiller, play is a force of civilization, which helps humans rise above their instincts and become members of enlightened communities ${ }^{[1]}$ (" humans are only fully human when they play"). The Dutch historian Huizinga ( founder of modern cultural history), saw games as a starting point for complex human activities such as language, law, war, philosophy and art.

Educational Games are games that have been specifically designed to teach people about a certain subject, expand concepts, reinforce development, understand an historical event of culture, or assist them in learning a skill as they play. This includes board, card and video games. Just a few places you see Educational games being used are: K-12, Universities, Military, Business. Officially games were incorporated in education early in the 19th century, with the creation of Kindergarten by Friedrich Fröbel, which was based on learning through simple educational toys such as blocks, sewing kits, clay, and weaving materials.

\section{Study Case I: the board game "StarStorm, The Galaxy Domination"}

\subsection{Description of the game}

The game "StarStorm" was created as an entry to the National Hellenic Contest of Astronomy to celebrate the 2009 IYA, run by the Hellenic Physical Society, where it won the second place. The team that created the board game was a school team of mine that consisted of 8 students. We carefully designed the game based on the following features : align game goals with cognitive work, uncertainty of outcome agreed upon rules with hard competition, make it adaptive, build in opportunities for suspense, conflict, and complication, make sure the game is simple in the right way, separate place and time, elements of fiction, elements of chance and personal enjoyment ${ }^{[6]}$.

The general objective of the created board game is to promote students/public understanding of science and astronomy, that is by playing. The "StarStorm" board game engages the game players from I. 10-18 years old, to activate their personality on the intellectual, the emotional, the desire, the intuitive and the imaginative level, and, II, older than 19 years, to discover the wonders of the Universe, the myths and basic characteristics of the constellations. Additionally, "StarStorm" gives players the message that it is not only luck (dice) but it is also scientific knowledge (question and cosmic events cards) which makes a civilization to survive.

In detail, "StarStorm" is a strategic board game for 4 players. It is played on a board depicting a constellation map, divided into territories-constellations. Players are in command of an army fighting for galaxy domination. They have to organize their forces to crush their enemies and dominate the constellations in this fast-paced game of strategy, negotiation, knowledge, and luck. It's up to them to deploy their troops, attack their enemies, and even betray their allies, in an aggressive effort to find a habitable exoplanet, as their new home, since they cannot live on their planet anymore because they have contaminated it. Through this game the players (from ages 10 to 100!!), are encouraged and motivated with a pleasant and entertaining way to get astronomical knowledge, through the cards of the game, and familiarize themselves with the night-sky constellations and 
learn about them from the board itself. For the game we created 1) a leaflet with the rules , 2) CONSTELLATION cards, which include a brief description of the respective constellation remarking the main features and the myths beyond them, and its pattern, 3) MISSION cards, with the mission that is given to each player for the game, and, 4) QUESTION cards and COSMIC FACT cards that have been carefully created and are related to edge-on astronomy topics. For the area (constellation) movement, "StarStorm" ignores realistic limitations, using the technology of wormholes!!

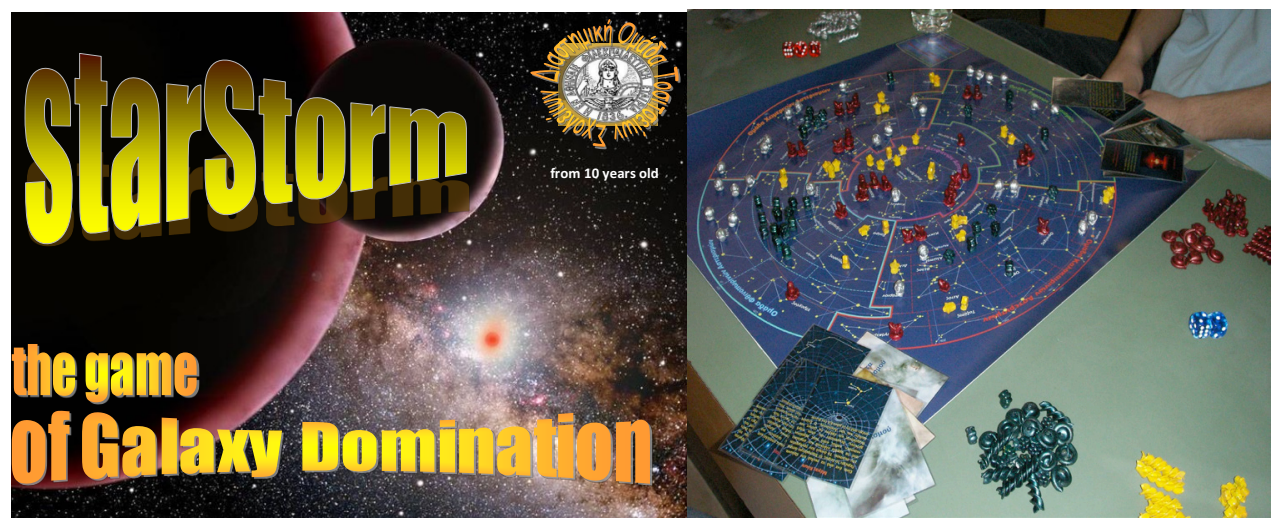

Fig.1 The board game "StarStorm"

\subsection{The Evaluation}

Research shows that games as a medium can be effective, but not always. Design is really what matters. Nobody assumes that all lectures, labs or books are good simply because of their medium ${ }^{[7,8]}$. We thus synthesize comparisons of game conditions versus non-game conditions (i.e., media comparisons) correlated with multiple learning objectives, requiring only basic understanding of required skills in order to evaluate our game.

For the evaluation, 50 students played the game. We carefully created a questionnaire and applied it as well also to 50 other students who did not play the game. The result was that when students played they adopted a mastery mindset that is highly conducive to learning. Moreover, students' interest and enjoyment increased when they played.

Additionally:

- 30 gymnasium students found that the game was more motivating than pencil and paper activities when learning astronomy (motivation and conceptual learning increased).

- 20 lyceum students found that using the board game was significantly better than formal learning (of the constelllations etc).

As well, students claimed that by playing the game, they did not need guidance, did not need to be challenged and did not need time to reflect!

\section{Study case II: Learning about the Galaxy by constructing a Galactic Garden at school}

In this gamification project we designed a flower replica of our Galaxy! Most bright stars in our Milky Way Galaxy reside in a disk. Since our Sun also resides in this disk, these stars appear to us as a diffuse band that circles the sky. The above panorama of a northern 
band of the Milky Way's disk covers 90 degrees. Visible are many bright stars, dark dust lanes, red emission nebulae, blue reflection nebulae, and clusters of stars. All the above and more can easily be explained and tought to students by creating a galactic garden at school, with them. The aim, was to engage students in the learning process by gamification. The procedure we followed was to learn about a) galactic coordinates, b) the structure of the galaxy, then set up the scale of the model to "plant" and decide about the content to be demonstrated and carefully choose the plants/flowers that will correspond to the content and make up the budget! Then work for the creation and walk around this Galactic Garden asking and answering questions.

All the data used were taken by the 2008c-10b map of NASA/JPL- Caltech /R.Hurt. We didn't evaluate this project, but students always enjoy walking, asking and learning through the galactic-garden about the Galaxy.

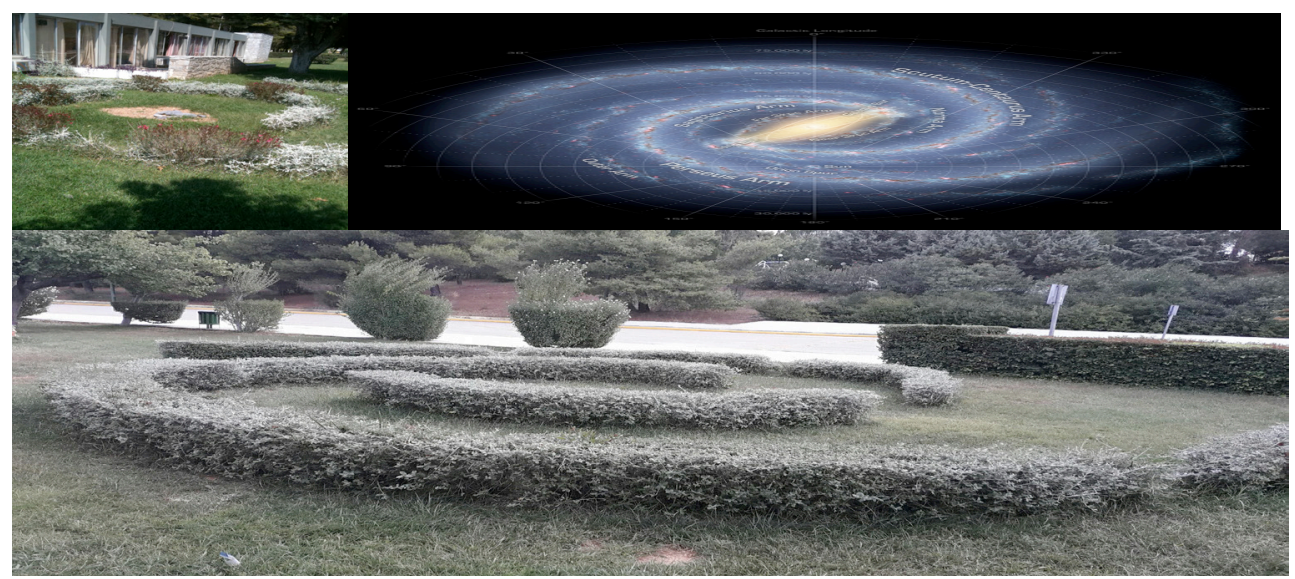

Fig.2 The Galactic Garden.

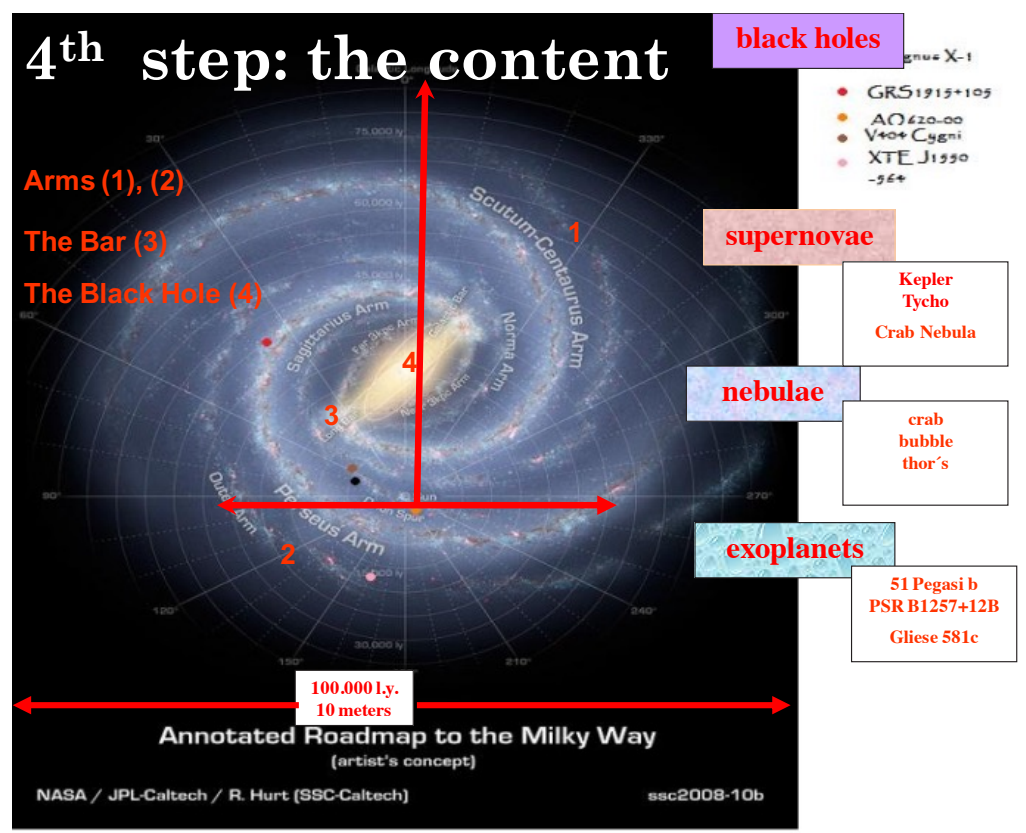

Fig.3 The content of our Galactic Garden. 


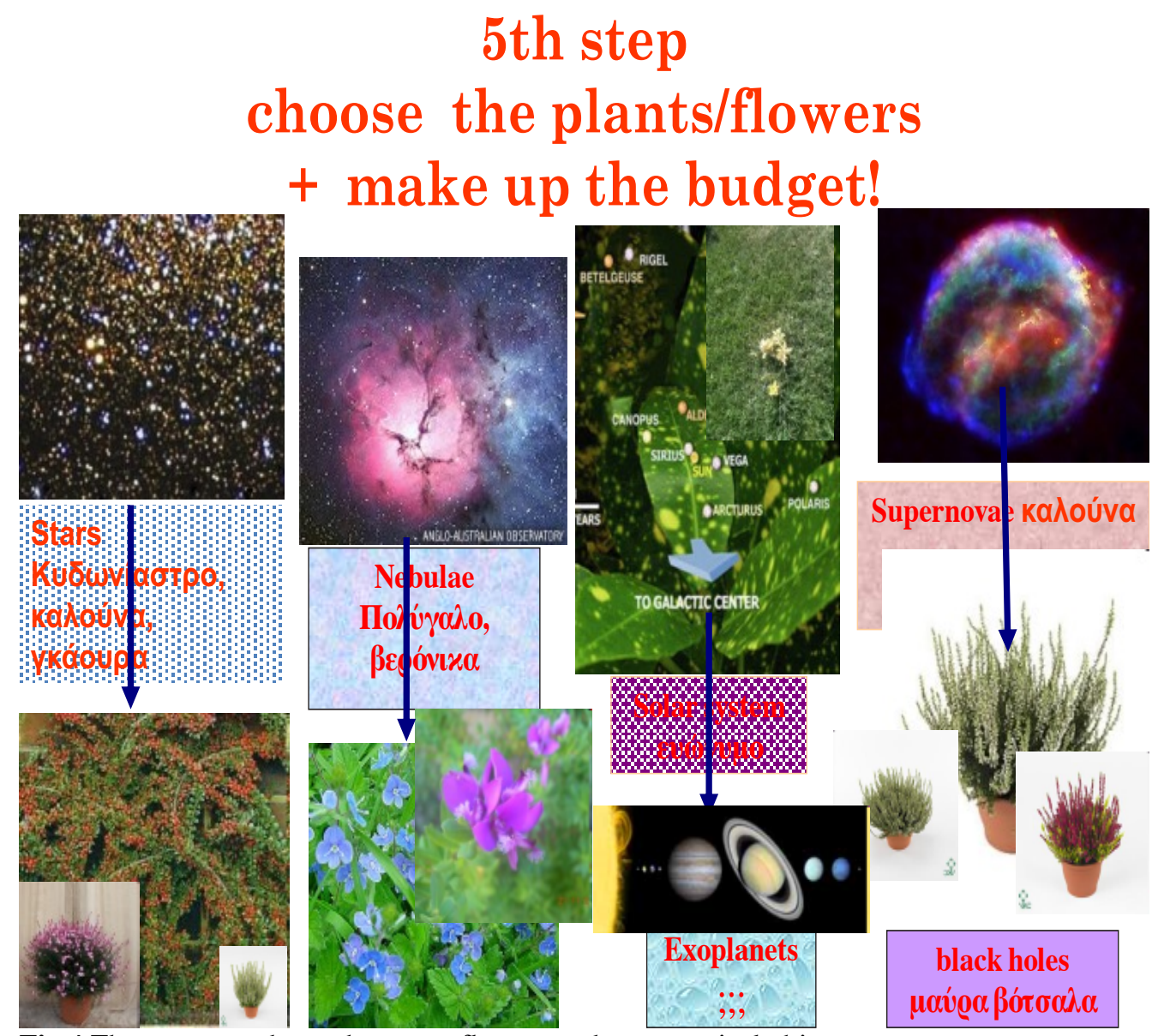

Fig.4 The correspondence between flowers and astronomical objects

\section{Conclusion}

Collective intelligence is a good practice in the service of pooling knowledge and diversity to make the world a better place and is applied to games as architectures for engagement. Nevertheless without a strong evidence base to back up games' academic effectiveness, the allure of using games for learning is hard to pass up. As research evolves, ensuring good assessment practices is critical for ensuring effective learning in games. A set of criteria is needed to help sort through all of this, and future research will need to examine design features that optimize learning across curricula.

Students played the "StarStorm" game and entered the gamification procedure of creating a Galactic Garden, got thrilled by the experience! Astronomy is a unique opportunity for implementing "New Literacies", which expands the conception of literacy beyond books and reading and through it to introduce science to students.

\section{References}

1. F.Schiller, ed. \& tr. E. M. Wilkinson \& L. A. Willoughby, Oxford: Clarendon Press, (1967) 
2. R. Aumann, S. Hart, Handbook of Game Theory with Economic Applications v. 1, ch. 3-6 and v. 3, ch. 43, (1992, 2002)

3. M.Metaxa, Innovation in Astronomy education, p.85, ed. J.M.Pasachoff, R.M.Ros, IAU GA in Prague(2006)

4. M.Metaxa, Advances in Hellenic Astronomy During the IYA09, ASP Vol.424, p.483, Athens, 2009

5. J. C. Glenn, Finnish Society for Futures Studies, Helsinki, Finland, One of the Next Big Things, Futura 4(2009)

6. A.Koch, J. Nafziger, H. Skyt Nielsen, J. of Economic Behavior \& Organization Vol. 115, Pages 3-17, (2015)

7. P.Sengupta, K.Krinks, B. Clark, J. of the Learning Sciences, 24(4), 638-674, (2015)

8. D. B., Clark, E.Tanner-Smith, S. Killingsworth, Review of Educational Research, 86(1), 79-122, (2016). 